\title{
Personalized Genomes and Cardiovascular Disease
}

\author{
Kiran Musunuru ${ }^{1,2}$ \\ ${ }^{1}$ Division of Cardiovascular Medicine, Brigham and Women's Hospital, Boston, Massachusetts 02115 \\ ${ }^{2}$ Department of Stem Cell and Regenerative Biology, Harvard University, Cambridge, Massachusetts 02138 \\ Correspondence: kiranmusunuru@gmail.com
}

The emerging ability to obtain a personalized genome, that is, to perform whole-genome sequencing to determine the entirety of the DNA sequence in an individual patient's chromosomes, holds out the promise of transforming patient care by allowing physicians to more accurately predict the risk of disease and to tailor therapy to that individual. Although no established applications of personalized genomics in cardiovascular medicine yet exist, there are at least two emerging applications that may ultimately become everyday practice. In the first application, DNA sequence variants that have been found to be associated with cardiovascular disease may be incorporated in risk-prediction algorithms to more accurately forecast whether patients will develop disease. In the second application, known as pharmacogenomics, DNA sequence variants that have been found to be associated with either beneficial effects or adverse effects of a medication may be used to help decide which medications or dosages of medications to prescribe to patients. It remains to be seen whether either of these applications will prove to be both cost effective as well as of clinical benefit.

$\mathrm{R}^{\mathrm{s}}$ ecent advances in technology have made iit possible to efficiently characterize genetic variation in large numbers of individuals. Genotyping arrays now have the capacity to accurately determine the identity of millions of common DNA sequence variants in an individual sample of DNA in a single experiment. Next-generation sequencing platforms have gone a step further in being able to sequence the entirety of the genome and thus determining the identity of most of the six billion DNA base pairs in an individual human genome in a matter of days. These techniques are making it possible to study cohorts of up to hundreds of thousands of individuals and determine which genetic variants influence the incidence of cardiovascular disease and the efficacy and safety of treatments for disease.

Risk-prediction algorithms, such as the Framingham risk score, are routinely used to prognosticate whether particular healthy patients are more (or less) likely to develop cardiovascular disease in the future. Partly based on those predictions, physicians choose whether to prescribe preventative medications, such as aspirin and a statin drug. The algorithms, which typically include traditional cardiovascular risk factors, such as age, sex, tobacco use, cholesterol levels, and blood pressure, are far from perfect, and considerable effort has been devoted to incor-

Editors: Margaret Buckingham, Christine L. Mummery, and Kenneth R. Chien

Additional Perspectives on The Biology of Heart Disease available at www.perspectivesinmedicine.org

Copyright (C) 2015 Cold Spring Harbor Laboratory Press; all rights reserved; doi: 10.1101/cshperspect.a014068

Cite this article as Cold Spring Harb Perspect Med 2015;5:a014068 


\section{K. Musunuru}

porating nontraditional risk factors into the algorithms to improve their predictive power. As successively larger human genetic studies identify increasing numbers of genetic variants that are associated with cardiovascular disease, efforts are underway to integrate this new genetic information into risk-prediction algorithms in the hopes that it will help better discriminate between those people who will develop disease and those who will not. If that proves the case, then one can anticipate personalized genomes becoming a standard element of risk prediction.

Pharmacogenomics, the study of variations of DNA and RNA characteristics as related to drug response, has emerged as a particularly promising area for the clinical application of personalized genomes. Commonly used cardiovascular medications, such as lipid-lowering drugs, antiplatelet agents, anticoagulants, and antiarrhythmic drugs, have been shown to have differential effects in people depending on whether they harbor particular genetic variants. The ultimate objective of pharmacogenomics is the use of "the right dose of the right drug for the right patient" by predicting the therapeutic response to the drug, as well as any adverse consequences from the drug, before the drug is prescribed.

Herein we will review the state of the science in human genetics and the techniques that are being used to identify DNA sequence variants associated with disease and therapeutic outcomes, early attempts to use genetic information to improve cardiovascular risk-prediction algorithms, and emerging pharmacogenomic applications that may translate to the clinic in the next few years.

\section{GENOME-WIDE ASSOCIATION STUDIES AND NEXT-GENERATION SEQUENCING STUDIES}

The human genome is roughly six billion DNA base pairs in size, with most of those base pairs being identical across all members of the human species. What marks each individual as unique are DNA sequence variants distributed throughout the genome. Some of these DNA sequence variants are quite common, occurring in many individuals in the general population and, in some cases, the majority of the people in a population. Other DNA sequence variants are rare and may be found in just one individual or family. Together, the full complement of variants may influence a particular individual's predisposition to (or protection against) cardiovascular disease, as well as that individual's response (whether favorable or adverse) to medications intended to treat cardiovascular disease.

Common DNA sequence variants tend to have subtle, if any, effects on gene function and, by extension, minimal effects on reproductive fitness; typically, any common variants with major phenotype consequences would have either been subjected to negative selection pressure and bred out of the population or subjected to positive selection pressure and spread universally throughout the population. (Exceptions include the $\beta$-hemoglobin gene-coding variant responsible for sickle cell anemia, which is believed to have persisted as a common variant in the population because it also provides protection against malaria infection when present in the heterozygous state, thus creating a balance between positive and negative selection.) In contrast, spontaneously arising rare DNA sequence variants may have severe phenotypic consequences and thus cause monogenic diseases in a few individuals. Although such variants might not persist in a population in the long term, the individuals bearing the variants may have sufficient longevity that extensive resources will be devoted to their health care over their lifetimes.

Genome-wide association studies (GWAS) are a methodology that emerged during the decade after the completion of the Human Genome Project and the first cataloging of common DNA sequence variants in human populations. GWAS use genotyping arrays that simultaneously determine the identities of millions of common DNA sequence variants across the genome, with the intent of detecting differences in the frequencies of individual variants in patients with a disease compared with healthy patients; variants with differing frequencies are construed as being associated with the disease and as marking a locus in the genome where either a gene or a regulatory element acts in a 
causal fashion to influence the pathogenesis of the disease. Although gaining an understanding of which genes are causal and the mechanisms by which they influence disease can require extensive experimentation (and is beyond the scope of this article), the common DNA sequence variants associated with the disease can potentially be used to predict which individuals are at greater or lesser risk for developing the disease. Similarly, common DNA sequence variants associated with a beneficial or adverse response to a medication can potentially be used to determine whether the medication is an optimal choice for a patient.

Rare DNA variants that cause disease are much more challenging to identify. Traditionally, the linkage study has been used in a family or families with an inherited pattern of disease to pinpoint the genomic locus of the disease gene, but even in the best-case scenario, DNA sequencing of candidate genes in the locus is required to identify the disease-causing variant or variants (typically coding variants). During the past few years, next-generation DNA sequencing technology has made it possible to rapidly and affordably sequence all of the coding regions in the genome ("exome sequencing") and, even more recently, the entirety of the genome ("whole-genome sequencing"). Although it may seem that such technology should make it straightforward to identify rare DNA variants causing disease, it has emerged that each individual has thousands of rare DNA variants, and there is no easy way to predict the functional consequences of any of those variants, not to mention identify the one variant that is responsible for the disease in that individual. At best, one can make an educated guess based on whether a rare variant is found in a gene that has previously been implicated in the disease in other individuals or families.

Next-generation sequencing is being used to study large numbers of individuals with extreme phenotypes in the population. The rationale is that genes that are found to have an assortment of rare variants in individuals with severe disease but few rare variants in healthy individuals (or, conversely, an assortment of rare variants in individuals who are protected against disease but few rare variants in individuals with disease) are important contributors to the disease. Thus far, such studies have been limited to a few thousand individuals each because of the expense of next-generation sequencing, and it has become clear that the studies are underpowered to discover new genes. Success awaits the sequencing of tens of thousands or even hundred of thousands of individuals.

With respect to predicting which individuals are likely to develop a disease or have a desirable response to a medication, common DNA sequence variants are more likely to be useful in the foreseeable future. At the same time, because common variants usually have small effects on gene function and clinical phenotypes, each individual variant's utility may be limited (see below). The individual variants may be more useful for the identification of novel genes and molecular pathways involved in diseases, because an improved understanding of pathobiology may pave the way for the development of new therapeutics. In contrast, next-generation sequencing is likely to be of little use for the typical person, owing to the difficulty in interpreting a rare variant that is private to that person, whereas it may be useful as a complement to linkage analysis in a family with an inherited pattern of disease (e.g., with an autosomal recessive pattern of inheritance).

\section{IMPROVING CARDIOVASCULAR RISK PREDICTION}

To date, GWAS have been applied to a variety of cardiovascular phenotypes. Among the most deeply characterized are blood lipid concentrations-low-density lipoprotein cholesterol (LDL-C), high-density lipoprotein cholesterol (HDL-C), and triglycerides-with more than 95 associated genomic loci identified to date (Teslovich et al. 2010), and coronary artery disease, with more than 30 associated loci identified to date (Coronary Artery Disease (C4D) Genetics Consortium 2011; Schunkert et al. 2011). For some of the loci, the causal gene and mechanism are well established, but the biological underpinnings of the majority of the loci remain to be explained. One caveat of 
these studies is that they were undertaken in cohorts of individuals of European descent, and so the identified common DNA variants may not be relevant to individuals of other ethnicities - an important limitation.

Conventional cardiovascular risk algorithms, such as the Framingham risk score, do not accurately predict many coronary events. As such, much effort has been aimed at identifying novel risk factors that, when combined with conventional risk factors, will better predict which individuals will develop disease. Not surprisingly, there is significant interest in the use of genetic information to improve risk prediction. In one of the first such studies, a genetic risk score combining nine common DNA sequence variants associated with either LDL-C or HDLC, was found to be associated with incident cardiovascular disease in a prospective cohort study of European individuals, with each unfavorable variant conferring a $15 \%$ increase in risk after adjustment for traditional risk factors, including plasma lipid concentrations (Kathiresan et al. 2008). Individuals with high genetic risk scores had a 63\% increased risk compared with individuals having low risk scores. However, addition of the genetic risk score to traditional risk factors did not significantly improve risk discrimination, with no change observed in a commonly used metric (C statistic). Two important limitations were that (1) all of the DNA sequence variants used in this genetic risk score predated the GWAS era, and so did not incorporate the most recent genetic discoveries; and (2) the DNA sequence variants were chosen because they were associated with blood lipid phenotypes rather than coronary artery disease-intermediate phenotypes rather than the clinical outcome of ultimate interest-which likely weakened the utility of the genetic risk score.

The first wave of GWAS for coronary artery disease identified a novel locus on chromosome 9 p21 as the strongest population-wide genetic risk factor for disease (Helgadottir et al. 2007; McPherson et al. 2007; Samani et al. 2007); this locus is not associated with any of the traditional risk factors for coronary artery disease, but it is associated with aortic abdominal aneurysm and intracranial aneurysm (Helgadottir et al.
2008), suggesting that the locus represents a hitherto unknown vascular risk factor. When considered on its own, the 9p21 genotype confers up to a $60 \%$ increased risk of coronary artery disease in individuals of European descent (McPherson et al. 2007; Talmud et al. 2008; Myocardial Infarction Genetics Consortium et al. 2009). Despite this promising result, attempts to incorporate a 9p21 DNA sequence variant into risk-prediction models have been disappointing. The addition of the 9p21 genotype to traditional risk factors in prospective cohort studies with individuals of European descent (Talmud et al. 2008; Brautbar et al. 2009; Paynter et al. 2009) resulted in no or minimal improvement in risk discrimination (as judged by the $\mathrm{C}$ statistic).

A comprehensive genetic risk score for coronary artery disease would include not just lipid-associated variants or the single best coronary artery disease-associated variant, but rather a collection of the most highly associated variants for disease. A risk score comprising 13 GWAS variants associated with coronary artery disease or myocardial infarction, including a 9p21 variant and four variants associated with blood lipids, yielded a $66 \%$ increase in risk for incident disease for individuals of European descent in the top quintile of the risk score compared with individuals in the bottom quintile; however, there was no improvement in the C statistic (Ripatti et al. 2010). Whether more comprehensive genotype scores incorporating all of the common DNA variants discovered to be associated with cardiovascular disease will perform better is now being evaluated. One important consideration is that having a family history of early-onset myocardial infarction in at least one parent more than doubles the personal risk of having a cardiovascular event (Lloyd-Jones et al. 2004). As more disease-associated variants are added to a comprehensive genetic risk score, it will be critical to assess whether the score adds any predictive value above and beyond simply asking an individual about his or her family history. It may well emerge that genetic risk scores have little value for most adult patients. Instead, genetic risk scores may prove most useful in children or 
young individuals to gauge lifetime cardiovascular risk and guide early interventions (socalled "primordial prevention").

\section{PHARMACOGENOMICS}

Pharmacogenomics focuses on the identification of DNA sequence variants that are associated with response to therapy. For a DNA sequence variant to be useful, patients with different genotypes of the variant should display significantly different responses to a medication-whether a therapeutic response or an adverse response. Typically, the variants in question are located in or near genes that encode enzymes that metabolize the medication. One genotype may result in increased (or decreased) activity of the enzyme compared with another genotype, resulting in different blood levels of the original medication or of an active metabolite. In some examples, there is no known biological link between the genetic variant and the medication, only a statistical association between the genetic variant and the patient response to the medication.

As of yet, there are not any widely accepted clinical uses of pharmacogenomics in cardiovascular medicine. However, there are three emerging applications-related to warfarin, clopidogrel, and statins - that may ultimately find routine use in patient care.

\section{Warfarin}

Warfarin, an anticoagulant widely used for the prevention and treatment of thromboembolic disease, is a challenging medication to use because of the highly variable responses among patients and even within an individual patient, with a number of factors influencing the responses including age, diet, weight, and use of interacting medications. Patients on warfarin require frequent monitoring of blood clotting activity as measured by the prothrombin timeinternational normalized ratio (INR), particularly in the first few weeks after initiation of the drug when the patient's ultimate stable therapeutic dosing is still being determined. There is significant risk of either bleeding if the dosing is too high or thromboembolism if the dosing is too low.

DNA sequence variants in two genes, CYP2C9 (cytochrome P450 2C9) and VKORC1 (vitamin $\mathrm{K}$ epoxide reductase complex subunit 1), have been shown to account for much of the interindividual variation in stable therapeutic dosing of warfarin (Wadelius et al. 2007; Gage et al. 2008; International Warfarin Pharmacogenetics Consortium et al. 2009). CYP2C9 encodes the hepatic enzyme responsible for converting warfarin into an inactive form. VKORC1 - the pharmacological target of warfarin-controls the function of an enzyme complex that produces the active form of vitamin K. An initial trial evaluated an algorithm that used the CYP2C9 and VKORC1 polymorphisms to predict an optimal starting warfarin dose for anticoagulation (Anderson et al. 2007). When compared with the usual practice (choosing a starting dose based on one's best clinical judgment), the pharmacogenomic algorithm did not change the number of out-of-range INRs during the initiation period, although it did reduce the numbers and sizes of dosing changes needed to achieve stable therapeutic dosing.

Several subsequently published small prospective clinical trials suggested that addition of genetic information could improve the safety and efficacy of warfarin initiation (e.g., number of out-of-range INRs, time to stable therapeutic dosing), but none were adequately powered to assess clinical outcomes (Caraco et al. 2008; Eckman et al. 2009; Huang et al. 2009). The Medco-Mayo Warfarin Effectiveness Study (MM-WES) was the first large warfarin genotyping study and was designed to test whether the use of genotype information would reduce the incidence of hospitalizations from adverse effects of warfarin, whether bleeding or thromboembolism (Epstein et al. 2010). This study was unique in that it took place within a community practice setting rather than within the context of a randomized prospective study with strict inclusion and exclusion criteria. About 900 patients who were initiating warfarin therapy submitted DNA samples for determination of CYP2C9 and VKORC1 genotypes. This information was given to their providers, and the 
K. Musunuru

patients were followed for 6 months. Historical controls from the same community practice setting but who had initiated warfarin therapy the previous year, numbering about 2700 , were used as the primary comparison group.

MM-WES reported a 31\% reduction of hospitalization in the genotyped patients compared with the control patients $(P<0.001)$, with a $28 \%$ reduction of hospitalization caused by bleeding or thromboembolism. This striking result notwithstanding, there are aspects of the study that can be fairly criticized (e.g., the lack of randomization and the use of historical rather than contemporaneous controls).

In two prospective randomized trials comparing a clinical algorithm for determining the dosing for warfarin initiation to a pharmacogenomic algorithm using CYP2C9 and VKORC1 genotypes, there were trends toward reduced clinically significant bleeds with the use of pharmacogenetic algorithms, although no significant differences in the percentage of time in the therapeutic INR range were observed (Kimmel et al. 2013; Verhoef et al. 2013). In another prospective randomized trial comparing a pharmacogenetic algorithm with a nonalgorithmic, loading-dose regimen, the pharmacogenetic algorithm yielded a significantly greater percentage of time in the therapeutic INR range and significantly fewer supratherapeutic INR measurements (Pirmohamed et al. 2013).

Although additional research studies of various designs will be needed to confirm that warfarin genotyping results in clinical benefit in a cost-effective manner, the early results suggest that anticoagulation could emerge as the first widely adopted pharmacogenomic application in cardiovascular medicine. However, the recent availability of alternative oral anticoagulants, such as dabigatran that have fixed dosing and do not require INR monitoring, could ultimately preempt the widespread use of a pharmacogenomic test for warfarin if the drug should fall into disfavor.

\section{Clopidogrel}

Another cardiovascular pharmacogenomic application for the prediction of response to ther- apy involves the antiplatelet agent clopidogrel, which is widely used in postacute coronary syndrome (ACS) care, particularly after percutaneous coronary intervention (PCI). Patients display variable responses to clopidogrel therapy, linked to the conversion of clopidogrel into its active metabolite by the hepatic cytochrome P-450 2C19 enzyme. A number of DNA sequence variants in the CYP2C19 gene encoding this enzyme have been identified, with some of the variants resulting in reduced function of the enzyme (Gurbel et al. 2003; Hulot et al. 2006; Angiolillo et al. 2007).

Three large studies of mostly post-ACS and/or post-PCI patients on clopidogrel therapy genotyped the CYP2C19 gene and identified at least one reduced-function variant in $~ 30 \%$ of individuals. In all three studies, carriers of reduced-function variants experienced significantly higher rates of cardiovascular death, myocardial infarction, and stroke (Collet et al. 2009; Mega et al. 2009; Simon et al. 2009). In one study, reduced-function variant carriers also displayed lower plasma levels of the active metabolite of clopidogrel (Mega et al. 2009), consistent with reduced activity of the cytochrome P-450 2C19 enzyme. These studies prompted the release of a "boxed warning" label for clopidogrel by the U.S. Food and Drug and Administration (FDA) indicating that individuals carrying two reduced-function CYP2C19 variants (termed "poor metabolizers") experience diminished effectiveness of the drug at standard dosing, and that alternative therapeutic strategies should be considered in these patients.

Subsequent studies have challenged the utility of CYP2C19 genotype data. One study that used data from participants in clinical trials that compared the effects of clopidogrel versus placebo on cardiovascular outcomes found that the relative risk reduction seen with clopidogrel treatment was similar for carriers of reducedfunction CYP2C19 variants and noncarriers (Paré et al. 2010). However, very few of the participants had undergone PCI with stent placement. A different pharmacogenetic study in which the majority of participants underwent PCI with stent placement found that carriers of reduced-function CYP2C19 variants experi- 
enced a higher event rate on clopidogrel than noncarriers within $30 \mathrm{~d}$ of initiation of therapy, although in the long term there was no difference in the event rate on clopidogrel (Wallentin et al. 2010). Together, these studies suggest that (1) the effect of reduced-function CYP2C19 variants is more relevant in the acute setting rather than the long term, and (2) CYP2C19 genotype testing may be more useful in higher-risk patients who have undergone PCI with stent placement.

To assess whether the latter is true, a metaanalysis of nine clopidogrel pharmacogenomic studies comprising mostly PCI patients was undertaken. This study of almost 10,000 participants taking clopidogrel found that carriers of reduced-function CYP2C19 variants experienced a $57 \%$ increase in risk of cardiovascular death, myocardial infarction, or ischemic stroke compared with noncarriers; with respect to the most serious complication of PCI, stent thrombosis, there was almost triple the risk of this outcome in reduced-function CYP2C19 variant carriers (Mega et al. 2010). In contrast, other meta-analyses that included studies with lower-risk patients did not find significant relationships between CYP2C19 genotype and clinical events (Bauer et al. 2011; Holmes et al. 2011). Unlike with warfarin, there have not yet been any published reports from large clinical trials assessing whether the use of CYP2C19 genotype data improves clinical outcomes, although such trials are now underway. Such studies will be needed to determine whether routine postACS genotyping of CYP2C19 will be cost effective and beneficial to patients. In the meantime, providers may wish to consider alternative therapy-that is, newer medications, such as prasugrel and ticagrelor-when treating post-PCI patients who are known to have reduced-function CYP2C19 variants.

\section{Statins}

Statins, used for the primary and secondary prevention of cardiovascular disease, are some of the most widely prescribed drugs in the world. As such, there is significant interest in identifying DNA sequence variants that help predict either the efficacy of or adverse effects from statins in individual patients. GWAS of individuals with statin-induced myopathy have identified a DNA sequence variant in the SLCO1B1 gene, which encodes an organic anion transporter that regulates the hepatic uptake of statin drugs that confer up to 17 times the risk of myopathy (SEARCH Collaborative Group et al. 2008). The large difference in relative risk (though not absolute risk, given the rarity of statin-induced myopathy) conferred by this DNA sequence variant suggests that a screening test could be helpful in predicting which patients are at risk of getting myopathy before they are started on statins (Voora et al. 2009). Clinical studies to assess this potential pharmacogenomic application are now underway.

\section{CONCLUDING REMARKS}

As should be evident from this article, the application of personalized genomes to the prevention and treatment of cardiovascular disease remains in rapid evolution. It seems likely that genetic data will prove to be more useful in pharmacogenomic applications than in cardiovascular risk prediction, at least in the near future, but further studies are clearly needed before genetic data should be incorporated into cardiovascular clinical practice in any context. One interesting consideration is the timing of obtaining genetic information from patients. As things stand now, one of the impediments to the use of genetic data is the need to obtain the data at the point of care, which entails costs and delays that may reduce the cost effectiveness of the application of the genetic data to the point that it is prohibitive. However, with the cost of whole-genome sequencing falling below several thousand U.S. dollars per sample (at the time of this writing), it may soon be that every patient will have his or her genome sequence determined routinely on admission to a hospital or even at the time of birth, and knowledge of that individual's every common and rare DNA sequence variant will be immediately available to a provider. As cardiovascular risk prediction becomes more sophisticated and new pharmacogenomic applications emerge, personalized 


\section{K. Musunuru}

genomes will undoubtedly play a significant role in everyday clinical practice.

\section{REFERENCES}

Anderson JL, Horne BD, Stevens SM, Grove AS, Barton S, Nicholas ZP, Kahn SF, May HT, Samuelson KM, Muhlestein JB, et al. 2007. Randomized trial of genotype-guided versus standard warfarin dosing in patients initiating oral anticoagulation. Circulation 116: 2563-2570.

Angiolillo DJ, Fernandez-Ortiz A, Bernardo E, Alfonso F Macaya C, Bass TA, Costa MA. 2007. Variability in individual responsiveness to clopidogrel: Clinical implications, management, and future perspectives. J Am Coll Cardiol 49: 1505-1516.

Bauer T, Bouman HJ, van Werkum JW, Ford NF, ten Berg JM, Taubert D. 2011. Impact of CYP2C19 variant genotypes on clinical efficacy of antiplatelet treatment with clopidogrel: Systematic review and meta-analysis. $B M J$ 343: $\mathrm{d} 4588$.

Brautbar A, Ballantyne CM, Lawson K, Nambi V, Chambless L, Folsom AR, Willerson JT, Boerwinkle E. 2009. Impact of adding a single allele in the 9p21 locus to traditional risk factors on reclassification of coronary heart disease risk and implications for lipid-modifying therapy in the Atherosclerosis Risk in Communities study. Circ Cardiovasc Genet 2: 279-285.

Caraco Y, Blotnick S, Muszkat M. 2008. CYP2C9 genotypeguided warfarin prescribing enhances the efficacy and safety of anticoagulation: A prospective randomized controlled study. Clin Pharmacol Ther 83: 460-470.

Collet JP, Hulot JS, Pena A, Villard E, Esteve JB, Silvain J, Payot L, Brugier D, Cayla G, Beygui F, et al. 2009. Cytochrome P450 2C19 polymorphism in young patients treated with clopidogrel after myocardial infarction: A cohort study. Lancet 373: 309-317.

Coronary Artery Disease (C4D) Genetics Consortium. 2011. A genome-wide association study in Europeans and South Asians identifies five new loci for coronary artery disease. Nat Genet 43: 339-344.

Eckman MH, Rosand J, Greenberg SM, Gage BF. 2009. Costeffectiveness of using pharmacogenetic information in warfarin dosing for patients with nonvalvular atrial fibrillation. Ann Intern Med 150: 73-83.

Epstein RS, Moyer TP, Aubert RE, O Kane DJ, Xia F, Verbrugge RR, Gage BF, Teagarden JR. 2010. Warfarin genotyping reduces hospitalization rates results from the MM-WES (Medco-Mayo Warfarin Effectiveness study). J Am Coll Cardiol 55: 2804-2812.

Gage BF, Eby C, Johnson JA, Deych E, Rieder MJ, Ridker PM, Milligan PE, Grice G, Lenzini P, Rettie AE, et al. 2008. Use of pharmacogenetic and clinical factors to predict the therapeutic dose of warfarin. Clin Pharmacol Ther 84: 326-331.

Gurbel PA, Bliden KP, Hiatt BL, O'Connor CM. 2003. Clopidogrel for coronary stenting: Response variability, drug resistance, and the effect of pretreatment platelet reactivity. Circulation 107: 2908-2913.

Helgadottir A, Thorleifsson G, Manolescu A, Gretarsdottir S, Blondal T, Jonasdottir A, Jonasdottir A, Sigurdsson A,
Baker A, Palsson A, et al. 2007. A common variant on chromosome 9p21 affects the risk of myocardial infarction. Science 316: 1491-1493.

Helgadottir A, Thorleifsson G, Magnusson KP, Grétarsdottir S, Steinthorsdottir V, Manolescu A, Jones GT, Rinkel GJ, Blankensteijn JD, Ronkainen A, et al. 2008. The same sequence variant on 9p21 associates with myocardial infarction, abdominal aortic aneurysm and intracranial aneurysm. Nat Genet 40: 217-224.

Holmes MV, Perel P, Shah T, Hingorani AD, Casas JP. 2011. CYP2C19 genotype, clopidogrel metabolism, platelet function, and cardiovascular events: A systematic review and meta-analysis. JAMA 306: 2704-2714.

Huang SW, Chen HS, Wang XQ, Huang L, Xu DL, Hu XJ, Huang ZH, He Y, Chen KM, Xiang DK, et al. 2009. Validation of VKORC1 and CYP2C9 genotypes on interindividual warfarin maintenance dose: A prospective study in Chinese patients. Pharmacogenet Genom 19: 226-234.

Hulot JS, Bura A, Villard E, Azizi M, Remones V, Goyenvalle C, Aiach M, Lechat P, Gaussem P. 2006. Cytochrome P450 2C19 loss-of-function polymorphism is a major determinant of clopidogrel responsiveness in healthy subjects. Blood 108: 2244-2247.

International Warfarin Pharmacogenetics Consortium, Klein TE, Altman RB, Eriksson N, Gage BF, Kimme SE, Lee MT, Limdi NA, Page D, Roden DM, et al. 2009. Estimation of the warfarin dose with clinical and pharmacogenetic data. N Engl J Med 360: 753-764.

Kathiresan S, Melander O, Anevski D, Guiducci C, Burtt NP, Roos C, Hirschhorn JN, Berglund G, Hedblad B, Groop L, et al. 2008. Polymorphisms associated with cholesterol and risk of cardiovascular events. $N$ Engl J Med 358: $1240-1249$.

Kimmel SE, French B, Kasner SE, Johnson JA, Anderson JL, Gage BF, Rosenberg YD, Eby CS, Madigan RA, McBane $\mathrm{RB}$, et al. 2013. A pharmacogenetic versus a clinical algorithm for warfarin dosing. N Engl J Med 369: 2283-2293.

Lloyd-Jones DM, Nam BH, D'Agostino RB Sr, Levy D, Murabito JM, Wang TJ, Wilson PW, O’Donnell CJ. 2004. Parental cardiovascular disease as a risk factor for cardiovascular disease in middle-aged adults: A prospective study of parents and offspring. JAMA 291: 2204-2211.

McPherson R, Pertsemlidis A, Kavaslar N, Stewart A, Roberts R, Cox DR, Hinds DA, Pennacchio LA, TybjaergHansen A, Folsom AR, et al. 2007. A common allele on chromosome 9 associated with coronary heart disease. Science 316: 1488-1491.

Mega JL, Close SL, Wiviott SD, Shen L, Hockett RD, Brandt JT, Walker JR, Antman EM, Macias W, Braunwald E, et al. 2009. Cytochrome p-450 polymorphisms and response to clopidogrel. N Engl J Med 360: 354-362.

Mega JL, Simon T, Collet JP, Anderson JL, Antman EM, Bliden K, Cannon CP, Danchin N, Giusti B, Gurbel P, et al. 2010. Reduced-function CYP2C19 genotype and risk of adverse clinical outcomes among patients treated with clopidogrel predominantly for PCI: A meta-analysis. JAMA 304: 1821-1830.

Myocardial Infarction Genetics Consortium, Kathiresan S, Voight BF, Purcell S, Musunuru K, Ardissino D, Mannucci PM, Anand S, Engert JC, Samani NJ, et al. 2009. Genome-wide association of early-onset myocardial in- 
farction with single nucleotide polymorphisms and copy number variants. Nat Genet 41: 334-341.

Paré G, Mehta SR, Yusuf S, Anand SS, Connolly SJ, Hirsh J, Simonsen K, Bhatt DL, Fox KA, Eikelboom JW. 2010. Effects of CYP2C19 genotype on outcomes of clopidogrel treatment. N Engl J Med 363: 1704-1714.

Pirmohamed M, Burnside G, Eriksson N, Jorgensen AL, Toh $\mathrm{CH}$, Nicholson T, Kesteven P, Christersson C, Wahlström B, Stafberg C, et al. 2013. A randomized trial of genotypeguided dosing of warfarin. N Engl J Med 369: 2294-2303.

Ripatti S, Tikkanen E, Orho-Melander M, Havulinna AS, Silander K, Sharma A, Guiducci C, Perola M, Jula A, Sinisalo J, et al. 2010. A multilocus genetic risk score for coronary heart disease: Case-control and prospective cohort analyses. Lancet 376: 1393-1400.

Samani NJ, Erdmann J, Hall AS, Hengstenberg C, Mangino M, Mayer B, Dixon RJ, Meitinger T, Braund P, Wichmann HE, et al. 2007. Genomewide association analysis of coronary artery disease. N Engl J Med 357: 443-453.

Schunkert H, König IR, Kathiresan S, Reilly MP, Assimes TL, Holm H, Preuss M, Stewart AF, Barbalic M, Gieger C, et al. 2011. Large-scale association analysis identifies 13 new susceptibility loci for coronary artery disease. Nat Genet 43: 333-338.

SEARCH Collaborative Group, Link E, Parish S, Armitage J, Bowman L, Heath S, Matsuda F, Gut I, Lathrop M, Collins R. 2008. SLCO1B1 variants and statin-induced myopathy-A genomewide study. N Engl J Med 359: 789-799.

Simon T, Verstuyft C, Mary-Krause M, Quteineh L, Drouet E, Méneveau N, Steg PG, Ferrières J, Danchin N, Becquemont L, French Registry of Acute ST-Elevation and Non-ST-Elevation Myocardial Infarction (FAST-MI) Investigators. 2009. Genetic determinants of response to clopidogrel and cardiovascular events. $N$ Engl J Med 360: 363-375.

Talmud PJ, Cooper JA, Palmen J, Lovering R, Drenos F, Hingorani AD, Humphries SE. 2008. Chromosome 9p21.3 coronary heart disease locus genotype and prospective risk of CHD in healthy middle-aged men. Clin Chem 54: 467-474.

Teslovich TM, Musunuru K, Smith AV, Edmondson AC, Stylianou IM, Koseki M, Pirruccello JP, Ripatti S, Chasman DI, Willer CJ, et al. 2010. Biological, clinical and population relevance of 95 loci for blood lipids. Nature 466: 707-713.

Verhoef TI, Ragia G, de Boer A, Barallon R, Kolovou G, Kolovou V, Konstantinides S, Le Cessie S, Maltezos E, van der Meer FJ, et al. 2013. A randomized trial of genotype-guided dosing of acenocoumarol and phenprocoumon. N Engl J Med 369: 2304-2312.

Voora D, Shah SH, Spasojevic I, Ali S, Reed CR, Salisbury BA, Ginsburg GS. 2009. The SLCO1B1*5 genetic variant is associated with statin-induced side effects. J Am Coll Cardiol 54: 1609-1616.

Wadelius M, Chen LY, Eriksson N, Bumpstead S, Ghori J, Wadelius C, Bentley D, McGinnis R, Deloukas P. 2007. Association of warfarin dose with genes involved in its action and metabolism. Hum Genet 121: 23-34.

Wallentin L, James S, Storey RF, Armstrong M, Barratt BJ Horrow J, Husted S, Katus H, Steg PG, Shah SH, et al. 2010. Effect of CYP2C19 and $A B C B 1$ single nucleotide polymorphisms on outcomes of treatment with ticagrelor versus clopidogrel for acute coronary syndromes: A genetic substudy of the PLATO trial. Lancet 376: 1320 1328. 


\section{$\&_{\mathrm{CSH}}^{\infty} \&$ Cold Spring Harbor

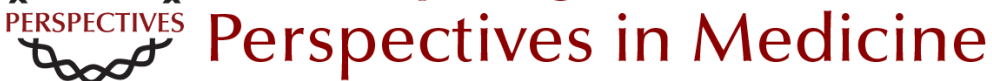

\section{Personalized Genomes and Cardiovascular Disease}

Kiran Musunuru

Cold Spring Harb Perspect Med 2015; doi: 10.1101/cshperspect.a014068 originally published online September 25, 2014

\section{Subject Collection The Biology of Heart Disease}

The Genetic Basis of Aortic Aneurysm Mark E. Lindsay and Harry C. Dietz

\section{Personalized Genomes and Cardiovascular \\ Disease \\ Kiran Musunuru}

Complex Genetics and the Etiology of Human

Congenital Heart Disease

Bruce D. Gelb and Wendy K. Chung

Genetic Networks Governing Heart Development Ashley J. Waardenberg, Mirana Ramialison, Romaric Bouveret, et al.

Heart Fields and Cardiac Morphogenesis Robert G. Kelly, Margaret E. Buckingham and Antoon F. Moorman

Regenerative Medicine: Transforming the Drug Discovery and Development Paradigm Sotirios K. Karathanasis

Myocardial Tissue Engineering: In Vitro Models Gordana Vunjak Novakovic, Thomas Eschenhagen and Christine Mummery

Pluripotent Stem Cell Models of Human Heart

Disease

Alessandra Moretti, Karl-Ludwig Laugwitz, Tatjana Dorn, et al.
Cardiac Cell Lineages that Form the Heart Sigolène M. Meilhac, Fabienne Lescroart, Cédric Blanpain, et al.

Synthetic Chemically Modified mRNA (modRNA):

Toward a New Technology Platform for

Cardiovascular Biology and Medicine Kenneth R. Chien, Lior Zangi and Kathy O. Lui

Next-Generation Models of Human Cardiogenesis via Genome Editing Xiaojun Lian, Jiejia Xu, Jinsong Li, et al.

How to Make a Heart Valve: From Embryonic Development to Bioengineering of Living Valve

Substitutes Donal MacGrogan, Guillermo Luxán, Anita Driessen-Mol, et al.

Insights into the Genetic Structure of Congenital Heart Disease from Human and Murine Studies on Monogenic Disorders Terence Prendiville, Patrick Y. Jay and William T. $\mathrm{Pu}$

Cardiovascular Drug Discovery: A Perspective from a Research-Based Pharmaceutical Company G. Gromo, J. Mann and J.D. Fitzgerald

Genetics and Disease of Ventricular Muscle Diane Fatkin, Christine E. Seidman and Jonathan G. Seidman

Embryonic Heart Progenitors and Cardiogenesis Thomas Brade, Luna S. Pane, Alessandra Moretti, et al.

For additional articles in this collection, see http://perspectivesinmedicine.cshlp.org/cgi/collection/ 